\title{
EDITORIAL
}

\section{IN THE JULY, 2010 ISSUE OF CLINICS}

\author{
Mauricio Rocha e Silva \\ doi: $10.1590 / \mathrm{S} 1807-59322010000700001$
}

In this July, 2010 issue of CLINICS we highlight a contribution by ANDRAUS et al. who describe the effect $\mathrm{S}$-nitroso-N-acetylcysteine on the ischemia-reperfusion injury caused in the experimentally induced steatotic liver lesions in rats and found that S-nitroso-N-acetylcysteine effectively protects against ischemia reperfusion injury in the steatotic liver but was without effect in the normal liver.
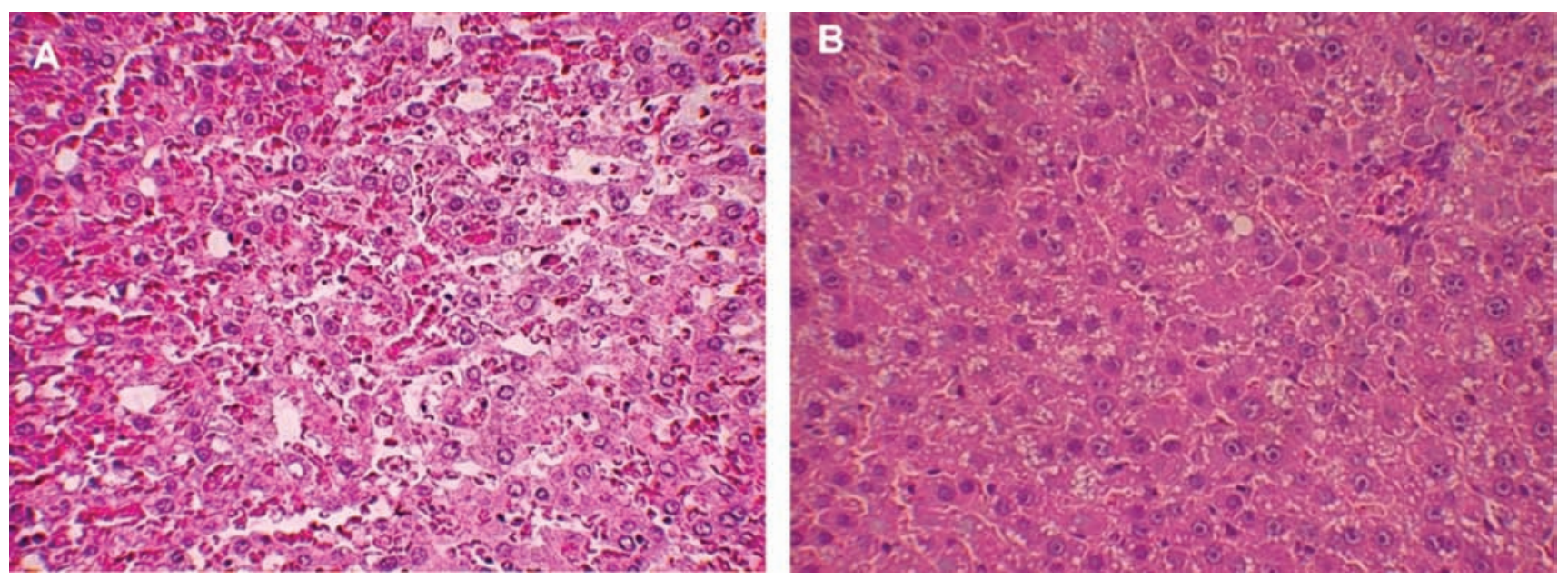

A. Steatotic, ischemic liver from a control rat (severe steatosis and hemorrhage). B. Steatotic, ischemic liver from an S-nitroso-N-acetylcysteine treated rat (moderate steatosis without hemorrahage).

We publish six CLINICAL SCIENCE REPORTS, two other BASIC RESEARCH ARTICLES AND 2 REVIEWS.

LACAZE et al. evaluated musculoskeletal discomfort and mental and physical fatigue in 64 call-center workers of an airline company before and after a supervised exercise program compared with rest breaks during the work shift and found that the discomfort decreased significantly for the spine and buttocks and the sum of the segments in the supervised exercise group. In addition, this group showed significant differences in the level of mental fatigue, especially in questions related to memory. They suggest that appropriately designed and supervised exercise programs

Hospital das Clínicas, Faculdade de Medicina da Universidade de São Paulo - São Paulo/SP, Brazil.

mrsilva36@hcnet.usp.br may be more efficient than rest breaks in decreasing discomfort and fatigue levels in call-center operators.

ROCHA et al. sought to establish an association between fluid and electrolyte balance in the first week of life and the risk of bronchopulmonary dysplasia through the clinical charts of 205 neonates. Clinical features, fluid and electrolyte balance were analyzed. They found a $22 \%$ prevalence of bronchopulmonary dysplasia associated to lower gestational age and birth weight, male gender, less frequent use of antenatal steroids, respiratory distress syndrome, use of surfactant, patent ductus arteriosus, duration of invasive ventilation and Neonate Intensive Care Unit stay. Serum values of potassium, phosphorus and creatinine during the first week of life also associated with bronchopulmonary dysplasia. They claim that differences in renal function and tubular handling of potassium and 
phosphorus are present during the first week of life among preterm neonates who will develop bronchopulmonary dysplasia.

FERREIRA et al. estimated the accuracy of the postural assessment software (PAS/SAPO) for measurement of corporal angles and distances as well as the inter- and intra-rater reliabilities using 88 pictures from 22 subjects; each subject was assessed twice (1 week interval) by 5 blinded raters. Inter- and intra-rater reliabilities were estimated using the intra-class correlation coefficient. They found that inter-rater reliability was excellent for $41 \%$ of the variables and very good for $35 \%$. Ten percent of the variables had acceptable reliability, and $14 \%$ were defined as non-acceptable. For intra-rater reliability, $44.8 \%$ of the measurements were considered to be excellent, $23.5 \%$ were very good, $12.4 \%$ were acceptable and $19.3 \%$ were considered non-acceptable. They found that unacceptable intra-class correlation coefficient values typically used the vertical line as a reference, and this may have increased the inaccuracy of the estimates. Increased accuracies were obtained by younger raters with more sophisticated computer skills, suggesting that past experience influenced results. They conclude that postural assessment software was accurate for measuring corporal angles and distances and should be considered as a reliable tool for postural assessment.

ANGELINI et al. compared the accuracy of tunnel placement and graft isometry for anterior cruciate ligament reconstruction performed using a computer-assisted navigation system (Orthopilot) and using traditional instruments. They conclude that the variation of the distance from the femoral to the tibial tunnel during flexion and extension was smaller in the Orthopilot group (better isometry) compared to the conventional group. There were no statistical differences in any other parameters between the groups, and all tunnels were considered to be in satisfactory positions.

PERINETTI et al. examined the correlation between dental malocclusion and body posture in 122 young subjects, all negative for temporo-mandibular disorders or other conditions affecting the stomatognathic systems, except malocclusion. Dental occlusion assessment was recorded through static posturography using a vertical force platform. They found that all of the posturographic parameters had large variability but were very similar between the two recording conditions. They conclude that their findings do not support existence of clinically relevant correlations between malocclusal traits and body posture.

CELIK et al. determined serum adenosine deaminase and xanthine oxidase levels in 30 patients with CrimeanCongo Hemorrhagic Fever and found a significant difference in adenosine deaminase and xanthine oxidase levels between cases and controls, even though neither adenosine deaminase nor xanthine oxidase levels varied with the severity of disease in the cases assessed. They also found that elevated serum xanthine oxidase activity in patients with CrimeanCongo Hemorrhagic Fever may be associated with reactive oxygen species generated by the xanthine/xanthine oxidase system during inflammatory responses and suggest that elevated lipid peroxidation may contribute to cell damage and hemorrhage. They also indicate that the association of cell damage and hemorrhage with xanthine oxidase activity should be further investigated in large-scale studies.

CUBAS et al. analyzed the amount of glycosaminoglycans in the uterine cervix during each phase of the rat estrous cycle and found that the highest levels of total sulfated glycosaminoglycans and dermatan sulfate occurred during the estrous phase. Heparan sulfate was prevalent during the proestrous phase and no significant variation in the concentration of hyaluronic acid in the uterine cervix was observed during the entire cycle. They suggest that the amount of total sulfated glycosaminoglycans may be influenced by hormonal fluctuations related to the estrous cycle, with dermatan sulfate and heparan sulfate being the glycosaminoglycans most sensitive to hormonal change.

UGUSMAN et al. studied the effect of Piper sarmentosum, a tropical plant with antioxidant and antiinflammatory activities, on nitric oxide production in oxidative stress using human umbilical vein endothelial cells and found that these endothelial cells treated with aqueous extract of $P$. sarmentosum showed a marked induction of Nitric oxide. They conclude that $P$. sarmentosum may improve endothelial function by promoting NO production in human umbilical vein endothelial cells.

FERREIRA FILHO et al. reviewed the theme of anti-hypertensive drugs and their differential effects on the regression of ventricular hypertrophy.

WIERNSPERGER et al. review the ever present controversy regarding the interaction of fructose and cardiometabolic disorders

\section{We also publish three case reports}

